\title{
The Efficacy of Digital Hearing Aids in the Management of Tinnitus in Individuals with Sensorineural Hearing Loss
}

\author{
Rajendra Kumar Porika ${ }^{*}$ \\ Balakrishnan Doraisami² \\ Aparna Ravichandran ${ }^{3}$
}

\begin{abstract}
Tinnitus is a condition which is often seen coexisting with hearing loss. In many persons with tinnitus, the use of amplification devices has been reported to show improvement in difficulties due to tinnitus. Though the underlying physiological mechanism is not clearly understood, hearing aids have proven beneficial. The aim of the study is to evaluate the benefit of the hearing aid in management of tinnitus. This study was conducted to assess whether such claims are true and, if so, what is the quantum of such benefit. In order to ascertain this, we studied the effects of three commonly used newer designs of digital programmable hearing aids namely, (i) Hearing aids with Basic programming, D-Basic (ii) those with tinnitus specific programming, DTS and (iii) those with in-built masking facility, DIM. In this study 108 subjects (65 males and 43 females), in the age range of 18 to 81 years were included. Each subject was fitted with one of the above mentioned three types of hearing aids, by qualified audiologists, purely on clinical grounds. All the subjects showed improvement in their hearing. The efficacy of the hearing aids, in mitigating the tinnitus, was assessed by employing the Tinnitus Handicap Inventory - THI. The THI has been developed by Newman et al in 1996 to study the effects of tinnitus, comprehensively under three domains viz. functional, emotional and catastrophic domains. A reduction in the THI scores indicates improvement. This tool is very popular and is acclaimed worldwide. It had been translated into several languages. In this study, the translated Telugu language version (THIT) of THI was used. The use of the local language (Telugu) afforded easy comprehension and better reliability. In each subject, we documented the THIT scores, before fitting of hearing aid and after two months of proper usage of the hearing aids. In the entire sample population of 108 subjects, across all the three different designtypes of hearing aids, we found a mean reduction of 42.6 points in the THIT scores. When the design of hearing aid was taken into reckoning, the mean post-fitting reduction of THIT scores in the subjects fitted with D-Basic, DTS, DIM hearing aids were 32.2, 43.5 and 51.9 respectively. In all the three designs, several subjects, those who were in a worse grade of tinnitus severity category of tinnitus severity before fitting, improved to a better grade after fitting. Further, we studied relief in the domain sub scales of the THIT viz. functional, emotional and the catastrophic domains. While all the three designs gave over-all relief of tinnitus, we found differences in the domain sub scales. However, designs with the In built maskers and tinnitus specific programming fared better. The conclusion drawn from our study is that, apart from the amplification benefit, all the three types of digital programmable hearing aids provided appreciable mitigation of tinnitus. Among the three design-types, hearing aids with inbuilt masker (DIM) were found to give the best benefit. Hearing aids with tinnitus specific programming (DTS) were the second best.
\end{abstract}

Keywords: Tinnitus; Digital Hearing aids; Programmable hearing aids; Tinnitus maskers; Tinnitus Handicap Inventory.

${ }^{1}$ Department of Audiologist and Speech Language Pathologist, Ali Yavar Jung National Institute of Speech and Hearing Disabilities (Divyangjan), Regional Centre, India ${ }^{2}$ Department of Audiology \& Speech Language Pathology, SRM Medical College Hospital and Research Centre, India

${ }^{3}$ Department of Audiologist and Speech Language Pathologist, Ali Yavar Jung National Institute of Speech and Hearing Disabilities (Divyangjan), India

Department of Audiologist and Speech Language Pathologist, Ali Yavar Jung National Institute of Speech and Hearing Disabilities (Divyangjan), Regional Centre, India E-mail: rajindrakumarp@gmail.com Phone: +919849236299

Paper submitted on February 08, 2021; and Accepted on May 20, 2021 


\section{INTRODUCTION}

The perception of sound in the ears in the absence of external stimulus is defined as tinnitus. A systematic review of the epidemiological studies worldwide indicates an overall prevalence of tinnitus ranging from $9 \%$ to $42.7 \%$. And, within these sufferers, the tinnitus was bothersome in $3 \%$ to $30.7 \%$. This wide range in the statistics can be attributed to differences in factors like definition of tinnitus, sample sizes, geographic locations etc ${ }^{1}$. But what is certain is that tinnitus is ubiquitous and has significant prevalence. Any amelioration of the distress would be quite welcome. For treatment of tinnitus, many options are now available. Tinnitus maskers, Tinnitus Retraining therapy etc. Tinnitus is frequently associated with sensorineural hearing loss. When a hearing aid is prescribed to an individual, with a primary objective of amplification, it has been found that several patients get relief from tinnitus also. Hearing aids are believed to mitigate tinnitus, in two ways, namely by providing additional auditory stimulation by the amplification of the environmental sounds and by masking the tinnitus sound itself. However, the actual mechanism which mitigates tinnitus is not yet definitively unraveled. Some recent advances in the design of hearing aids are claimed to improve this benefit. These newer type-designs provide environmental sounds, musical tones and other sounds. In recent times, an inbuilt tinnitus masking programme, has become available. This masker design targets the tinnitus, by matching the pitch and the intensity of the tinnitus. The audiologists have started utilizing this armamentarium of the newer type-designs, in increasing numbers. However, the issue of an evidence base is still lacking. The management of tinnitus begins with a detailed history. This is followed by Pure Tone Audiometry, Impedance audiometry, pitch and loudness matching of tinnitus, and residual inhibition tests. To quantify the impact of tinnitus in daily living, several questionnaire tools are available. A self-report tool called Tinnitus Handicap Inventory (THI) developed in 1996, by Newman et al is the most popular and is commonly used $^{2}$. It has 25 questions, which must be answered by the subjects themselves, with any one of three responses - namely 'Yes, Sometimes, or No' with numerical scores of 4, 2, 0 respectively. The 25 questions are arranged under three sub scales viz. Functional domain (12 questions), Emotional domain (8 questions), and Catastrophic domain (5 questions). The total maximum score is 100 . Higher scores represent greater handicap. Based on the THI score, four degrees of handicap are recognized - Mild, Moderate, Severe and Catastrophic categories. THI tool has high reliability and consistency ${ }^{3}$. THI score helps the clinician to tailor the treatment plan for the individual patient, and also to monitor the response. Over the years, the THI has been translated into many languages. In 2018, this tool was translated into Telugu language - $\mathrm{THIT}^{4}$. Telugu language is spoken by eight million people worldwide. Hence THIT is expected to find wide usage, in daily clinical practice.

Aim: The primary objective of this study was to evaluate the efficacy of digital programmable hearing aids of newer type-design, in mitigating tinnitus in subjects with coexisting tinnitus and sensorineural hearing impairment. For this purpose, three commonly used design-types were tested. The designs were (i) Digital programmable hearing aids with basic programming, D-Basic (ii) Digital programmable hearing aids with tinnitus specific programming on tinnitus, DTS and (iii) Digital programmable hearing aids with inbuilt tinnitus masking facility, DIM. The secondary objective was to compare their relative efficacy, in the subscales of tinnitus namely Functional, Emotional and Catastrophic domains.

\section{MATERIALS AND METHODS}

The design was observational, with tinnitus evaluation before and after fitting of newer designs of hearing aids. A total of 108 subjects suffering with tinnitus and co existing hearing loss were included in this study. They were aged above 18 years and were of both genders. We included only patients with severe or catastrophic grades of severity i.e. a THIT score of 58 and above. Regarding their hearing impairment, we ensured that they had at the least a moderate degree of sensorineural hearing loss. Patients suffering from external and middle ear disorders, chronic neurological disorders, and those who were already on some treatment for tinnitus were excluded. The hearing aid must have been fitted for the first time, during this study. The study was approved by the Institutional Ethics Committee of SRM University (1239/IEC/2017). Informed consent was obtained from all the subjects. Detailed case history, clinical examination, basic audiological tests, and speech audiometry were done. The profile of tinnitus namely duration, character, pitch and loudness were documented. The THIT was administered to obtain the effect of tinnitus on the subjects. One of the three design-types of hearing aids mentioned above, was fitted in the subjects, by qualified, experienced and registered audiologists. The appropriateness of the fitting was ensured by strict adherence to current standard practices and clinical guidelines. The patient and the servicing audiologist had full autonomy in the selection and fitting of the type of hearing aid, per requirement and affordability. All the subjects obtained hearing benefit from the amplification, as confirmed by Speech Identification Scores, Hearing in Noise test and by patient reported outcomes. Based on the type-design of the hearing aids fitted, the subjects were consecutively assigned into one of three groups, namely (i) D-Basic group (ii) DTS group and (iii) DIM group. When the number of subjects in each group, reached the required number of 36 , further recruitment into that group was stopped. There was no need for a control group, as this was a pre- and poststudy. Each subject was given complete instructions about the care of hearing aids. The subjects were advised to use the hearing aids, for a minimum of 2- 6 hours daily. After one month of usage, a follow-up visit was done, for monitoring proper use and compliance. After two months of regular use, the THIT scores were repeated. Statistical analysis was done using Statistical Package for the Social Sciences (SPSS) version 26, (year: 2017). Paired 't'-test 
was used for comparing pre- and post- THIT scores. ANOVA was used to evaluate the changes in the THIT scores in the case of each of the three groups. Chi-Square test was used to analyze the changes in the severity grades before and after fitting. During the study period, care was taken that the patient was not under any medication specific for tinnitus. However, other medicines prescribed for co-existing conditions like diabetes, hypertension etc, were continued. The only intervention for tinnitus was the wearing of the hearing aids. Absolute confidentiality and privacy were maintained by the investigators. Further, the subjects of this study did not intermingle and did not get to know the other subjects. No blinding between the subjects and the investigator was possible, because the very design of the study precludes such blinding.

\section{RESULTS}

The objectives of this study were to evaluate out whether the newer digital hearing aids, as a genre, were useful to mitigate the tinnitus and to ascertain which type-design offered better efficacy in mitigating tinnitus. A total of 108 subjects formed the study sample. Among them 65 were male, and 43 were female subjects. The age ranged from 18 - 81 years. The subjects reported tinnitus and hearing difficulty and on examination on were seen to have sensory neural hearing impairment of moderate degree. The subjects with severe and catastrophic degree of tinnitus were included in the study. For the purpose of this study, subjects fitted with one of the following three type-designs were recruited (i) Hearing aids with only Basic programming - D-Basic, (ii) Hearing aids with Tinnitus Specific Programming - DTS and (iii) Hearing aids with Inbuilt tinnitus Masking - DIM. Based on type of hearing aids used by them, 36 persons were assigned to each of three groups, namely D-Basic, DTS and DIM groups. The severity of tinnitus was recorded by the THIT tool, once at the time of fitting and a second time three months after fitting. The overall combined scores and also individual subscales viz. namely functional, emotional and catastrophic domains were recorded. The obtained data from the subjects were analyzed using Statistical Package for the Social Sciences (SPSS) version 26, year 2017), using appropriate tests such as Paired 't'-test, Analysis of Variance (ANOVA) and Chi-square tests.

\section{Improvements in the THIT scores in the entire sample, after fitting of hearing aids:}

The entire sample of this study ( $N=108)$, the total THIT scores (maximum 100 points) showed improvement after fitting of the hearing aids, irrespective of the design-type. The mean improvement in the total THIT scores, in the entire sample of 108 subjects (combined for all the three sub domains) was 42.6. This improvement was evident in each of the three sub scale domains also. Paired ' $T$ ' test showed the statistical mean differences in the THIT scores, to be $21,13.8$, and 7.6 respectively for the functional, the emotional and the catastrophic domains with ' $t$ ' values being statistically significant $(p<0.01)$ (Table 1$)$.

\section{Improvement in the THIT scores, in each of the three design-types of the Hearing aids:}

The benefits in the THIT scores were analyzed individually for each of the three design-type group viz. D-Basic, DTS and DIM. The results shows the mean difference of 32.2 ( $t$ $=8.7$ ) for the total THIT score for D-Basic group whereas for DTS group the mean difference was $43.5(t=13.9)$ and for DIM group it was $51.9(t=18.3)$ which are statistically significant. Further the scores were analyzed for each of the three domain and results showed for D-Basic hearing aids group the difference in means to $16.7(t=7.7), 10.9$ $(\mathrm{t}=8.9)$ and $4.6(\mathrm{t}=4.7)$ which are statistically significant and for DTS group the domain wise difference in means was $21.3(t=12.6), 13.3(t=12.6)$ and $8.4(t=10.3)$ whereas for DIM group the domain wise scores were 24.7 (15.0), 17.2 (19.5) and 10.0 (13.1) which are statistically significant. Hence, all the three groups when compared pre and post fitting showed statistical significance which can be inferred that all the three design - types mitigates tinnitus (Tables 2, 3 and 4). The Post Hoc analysis was done to check within group difference and the results as indicated in table 5, 6 and results revealed. The mean gain scores was calculated (Table 5) for overall domains was $32.2,43.5$, and 51.9 for three groups respectively and total THIT gain (improvement) of 42.5. Overall the F-ratio was 9.33 with significance of $0.01(p<0.01)$. The above Post Hoc (Table 6) gives the comparisons of between three groups D-Basic, DTS and DIM with respect to all domains combined. The mean gain scores obtained for all the three groups were compared using ANOVA test. ANOVA suggested that the mean difference between the scores was statistically significant with $p$-value equal to 0.01 which is $<0.05$ assumed for the study. Hence the Post Hoc analysis using Turkey HSD test was done to see which group comparison of the means showed significant difference among the groups. Test results showed significant difference between D-Basic and DTS with significant $p$-value 0.01 and $<0.05$ at $5 \%$ level of significance. The difference between D-Basic and DIM was also significant $p$-value 0.00 and $<0.05$, at $5 \%$ level of significance. The difference between DTS and DIM was not significant in population p-value 0.06 and $>0.05$ at $5 \%$ level of significance.

\section{Mitigation of the tinnitus by the hearing aids, analyzed by the categories of handicap}

The statistical analysis so far described, were based on the raw THIT scores. As a next step, the handicapping effect of tinnitus on subjects were classified into categories (based on the THIT scores). The four categories were (i) mild handicap category, (ii) moderate handicap category, (iii) severe handicap category and (iv) catastrophic handicap category. The subjects who completely relieved were classified into a 'no tinnitus' category (Newman et al, 1985). Out of the 108 total subjects, before fitting of the hearing aids, 57 persons were in the catastrophic category and 51 were in the severe category. After fitting, many of them shifted to better categories. This downshift 
Table 1. THIT scores in the entire study sample $(\mathrm{N}=108)$, before and after fitting - all the three domains combined and individually.

\begin{tabular}{ccccc} 
THIT scores in all three designs $(\mathrm{N}=108)$ & Total & \multicolumn{2}{c}{ Individual domains } \\
& (all 3 domains, combined) & Functional & Emotional & Catastrophic domain \\
domain only & domain only & only \\
Mean THIT score (SD) before fitting & $77.4(12.2)$ & $39.6(6.5)$ & $25.7(4.9)$ & $11.9(4.9)$ \\
Mean THIT score (SD) after fitting & $34.8(20.6)$ & $18.6(11.1)$ & $11.8(7.4)$ & $4.3(4.5)$ \\
Difference in the means & 42.6 & 21.0 & 13.8 & 7.6 \\
't' value (paired 't' test) & $21.2^{\star \star}$ & $18.9^{* \star}$ & $21.0^{* \star}$ & $14.4^{* *}$
\end{tabular}

Table 2: THIT scores in the D-Basic group $(\mathrm{N}=36)$, before \& after fitting - all domains combined and individual domain-wise.

\begin{tabular}{|c|c|c|c|c|}
\hline THIT scores in D-Basic design-type $(\mathrm{N}=36)$ & Overall THIT scores & $\begin{array}{l}\text { Functional } \\
\text { domain only }\end{array}$ & $\begin{array}{l}\text { Emotional } \\
\text { domain only }\end{array}$ & $\begin{array}{l}\text { Catastrophic domain } \\
\text { only }\end{array}$ \\
\hline Mean score (SD) before fitting & $81.8(8.4)$ & $41.6(5.0)$ & $27.7(3.4)$ & $12.4(5.3)$ \\
\hline Mean score (SD) after fitting & $49.6(19.9)$ & $24.8(12)$ & $16.8(7.5)$ & $7.8(4.3)$ \\
\hline Difference in the means & 32.2 & 16.7 & 10.9 & 4.6 \\
\hline 't' value, (paired T test) & $8.7^{\star \star}$ & $7.7^{\star \star}$ & $8.9 * \star$ & $4.7^{\star \star}$ \\
\hline
\end{tabular}

Table 3. THIT scores in the DTS group $(\mathrm{N}=36)$, before \& after fitting - all domains combined and individual domain-wise.

\begin{tabular}{|c|c|c|c|c|}
\hline $\begin{array}{l}\text { THIT scores in DTS group } \\
\text { design-type }(\mathrm{N}=36)\end{array}$ & Total & $\begin{array}{l}\text { Functional } \\
\text { Domain only }\end{array}$ & $\begin{array}{l}\text { Emotional } \\
\text { Domain only }\end{array}$ & Catastrophic domain only \\
\hline Mean score before fitting & $77.1(13.3)$ & $40.3(6.2)$ & $25.5(5.1)$ & $11.1(4.7)$ \\
\hline Mean score (SD) after fitting & $33.5(18.1)$ & $18.7(9.8)$ & $12.0(6.6)$ & $2.7(3.1)$ \\
\hline Difference in the means & 43.4 & 21.5 & 13.5 & 8.4 \\
\hline 't' value (paired T test) & $13.9 * *$ & $12.6^{\star *}$ & $12.6^{\star *}$ & $10.3^{* *}$ \\
\hline
\end{tabular}

Table 4. THIT scores in the DIM group $(\mathrm{N}=36)$, before \& after fitting - all domains combined and individual domain-wise.

\begin{tabular}{ccccc}
$\begin{array}{c}\text { THIT scores in DIM group } \\
\text { design-type (N=36) }\end{array}$ & Total & $\begin{array}{c}\text { Functional } \\
\text { domain only }\end{array}$ & $\begin{array}{c}\text { Emotional } \\
\text { domain only }\end{array}$ & Catastrophic domain only \\
\hline Mean score (SD) before fitting & $73.3(13.1)$ & $37.0(6.6)$ & $23.9(5.3)$ & $12.3(4.6)$ \\
Mean score (SD) after fitting & $21.3(12.7)$ & $12.3(7.7)$ & $6.7(4.0)$ & $2.3(3.7)$ \\
Difference in the means & 51.9 & 24.7 & 17.2 & 10.0 \\
't' value (paired T test) & $18.3^{\star *}$ & $15.0^{\star *}$ & $19.5^{\star *}$ & $13.1^{\star *}$
\end{tabular}

Table 5. Post fitting improvements in the THIT mean scores among the three hearing aid groups, combined for all the three subscales (all three domains).

\begin{tabular}{cccccc}
\hline Hearing aid group & N & Mean Scores & SD & F & $\begin{array}{c}\text { Significance } \\
(p \text {-value })\end{array}$ \\
D-Basic group & 36 & 32.2 & 22.0 & & \\
DTS group & 36 & 43.5 & 18.7 & 9.33 & 0.01 \\
DIM group & 36 & 51.9 & 16.9 & & \\
Overall & 108 & 42.5 & 20.8 & & \\
\hline
\end{tabular}

Table 6. Post Hoc analysis group wise multiple comparisons of improved THIT scores mean difference of all the subjects of the study $(\mathrm{N}=108)$ - Overall domains.

\begin{tabular}{cccc}
\hline Group (I) & Group (J) & Mean Difference (I-J) & $\begin{array}{c}\text { Significance } \\
(\mathrm{p} \text {-value })\end{array}$ \\
\hline \\
D-Basic & DTS & $-11.27^{\star}$ & 0.01 \\
DTS & DIM & $-19.6^{\star}$ & 0.00 \\
& D-Basic & $11.27^{\star}$ & 0.01 \\
DIM & DIM & -8.38 & 0.06 \\
& D-Basic & $19.66^{\star}$ & 0.00 \\
\hline
\end{tabular}

occurred in all the three design types. Three months after fitting of hearing aids, only five subjects were in the catastrophic category; and only 15 subjects were in the severe category (Table 7). Further, the downshift was analyzed for each of three design-types of hearing aids.
This enabled us to assess the trend of benefit with each design types.

D-Basic hearing aids: Among the 36 subjects fitted with this type-design, 12 subjects were in 'severe handicap' at the pre-fitting stage. Out of these 12 severe persons, four 
person's downshifted to mild category; five downshifted to moderate category; two subjects stayed in the same severe category itself. In this same group, 24 subjects were in 'catastrophic handicap' at the pre-fitting stage, of these nine downshifted to 'mild' category; four downshifted to moderate category; eight downshifted to severe category; unfortunately, three subjects showed no improvement at all and stayed in the catastrophic category itself.

DTS hearing aids: Among the 36 subjects fitted with this type-design, 19 subjects at the pre-fitting stage, were under 'catastrophic handicap' and on post fitting stage, two subjects downshifted to 'no tinnitus' category; nine subjects downshifted to mild category; four subjects downshifted to moderate category; three subjects downshifted to severe category. Unfortunately, one subject showed no improvement at all and stayed in the catastrophic category itself. Among 17 remaining subjects showed 'severe handicap category' at the prefitting stage. Out of these, four subjects downshifted to 'no tinnitus' category; nine subjects downshifted to mild category; three subjects downshifted to moderate category; one subject showed no improvement and stayed in the severe category itself at post fitting.

DIM hearing aids: Among the 36 subjects fitted with this type-design, 13 subjects were in 'catastrophic handicap category' at the pre-fitting stage, of which seven subjects downshifted to 'no tinnitus' category; four subjects downshifted to mild category; one subject downshifted to moderate category and one more subject downshifted to severe category. The remaining 23 subjects were in 'severe category' of handicap, in pre-fitting stage. Among them eight subjects downshifted to 'no tinnitus' category; fourteen subjects downshifted to mild category; one subject downshifted to moderate category (Table 6 and 7) on post fitting. From the Table 8 , it is observed that before fitting hearing aids, the Pearson Chi-square value was found to be 7.79 which is found to be not significant $(p>0.05)$. Hence, it can be inferred that there was no significant difference within each of the groups with respect to tinnitus severity grades before fitting

Table 7. The changes in the category of tinnitus handicap, in the entire sample i.e. combined for all the three design-types of hearing aids.

\begin{tabular}{ccc}
\hline Categories of Handicap & Before fitting Hearing aid & After fitting Hearing aid \\
\hline No tinnitus & 0 & $21(19 \%)$ \\
Mild & 0 & $49(45 \%)$ \\
Moderate & 0 & $18(16 \%)$ \\
Severe & $51(47 \%)$ & $15(13 \%)$ \\
Catastrophic & $57(52 \%)$ & $5(4 \%)$ \\
Chi-square & 7.79 & 35.23 \\
\hline
\end{tabular}

Table 8. Tabulation of the down shift in the categories of tinnitus handicap, after fitting of the three different design-types of hearing aids.

\begin{tabular}{|c|c|c|c|c|c|c|}
\hline \multirow[t]{2}{*}{$\begin{array}{l}\text { Categories of } \\
\text { Handicap }\end{array}$} & \multicolumn{3}{|c|}{ Before fitting } & \multicolumn{3}{|c|}{ After fitting } \\
\hline & D-Basic group (36) & DTS group (36) & DIM group (36) & D-Basic group (36) & DTS group (36) & DIM group (36) \\
\hline No tinnitus & 0 & 0 & 0 & 0 & $\begin{array}{c}6 \\
(16 \%)\end{array}$ & $\begin{array}{c}15 \\
(41 \%)\end{array}$ \\
\hline Mild & 0 & 0 & 0 & $\begin{array}{c}13 \\
(36 \%)\end{array}$ & $\begin{array}{c}18 \\
(50 \%)\end{array}$ & $\begin{array}{c}18 \\
(50 \%)\end{array}$ \\
\hline Moderate & 0 & 0 & 0 & $\begin{array}{c}9 \\
(25 \%)\end{array}$ & 7 (19\%) & $\begin{array}{c}2 \\
(5 \%)\end{array}$ \\
\hline Severe & $\begin{array}{c}12 \\
(33 \%)\end{array}$ & 17 (47\%) & $22(61 \%)$ & $\begin{array}{c}10 \\
(27 \%)\end{array}$ & $\begin{array}{c}4 \\
(11 \%)\end{array}$ & $\begin{array}{c}1 \\
(2 \%)\end{array}$ \\
\hline Catastrophic & $\begin{array}{c}24 \\
(66 \%)\end{array}$ & 19 (52\%) & $14(38 \%)$ & $\begin{array}{c}4 \\
(11 \%)\end{array}$ & $\begin{array}{c}1 \\
(2 \%)\end{array}$ & 0 \\
\hline
\end{tabular}

Table 9. Shifts in the handicap categories, after three months of hearing aid use, in each of the three hearing aid groups.

\begin{tabular}{|c|c|c|c|c|c|c|c|}
\hline \multirow{2}{*}{$\begin{array}{l}\text { Hearing aid } \\
\text { group }\end{array}$} & \multirow{2}{*}{$\begin{array}{c}\text { Pre fitting } \\
\text { Handicap category }\end{array}$} & \multirow{2}{*}{$\begin{array}{c}\text { No. of subjects } \\
\text { in the category } \\
\text { before fitting } \\
\text { hearing aid }\end{array}$} & \multicolumn{5}{|c|}{ No. of subjects in the severity category three months AFTER hearing aid fitting } \\
\hline & & & Catastrophic & Severe & Moderate & Mild & No tinnitus \\
\hline D-Basic & Severe & 12 & 1 & 2 & 5 & 4 & 0 \\
\hline$(\mathrm{N}=36)$ & Catastrophic & 24 & 3 & 8 & 4 & 9 & 0 \\
\hline DTS & Severe & 17 & & 1 & 3 & 9 & 4 \\
\hline$(N=36)$ & Catastrophic & 19 & 1 & 3 & 4 & 9 & 2 \\
\hline DIM & Severe & 23 & & & 1 & 14 & 8 \\
\hline$(\mathrm{N}=36)$ & Catastrophic & 13 & & 1 & 1 & 4 & 7 \\
\hline
\end{tabular}


hearing aids. It could be inferred that each group was homogenous in composition. In further analysis, the Post fitting THIT scores of each of the three hearing aid groups, namely D-Basic, DTS and DIM groups, as compared with the Pre-fitting scores. The respective scores in each of domains were also compared. From the table 9 it is observed that after fitting of hearing aids, the Pearson Chi-square value was 35.23 and found to be statistically highly significant $(p<0.01)$. Hence, it is inferred that there is a highly significant difference among the three groups with respect to tinnitus severity grades after fitting hearing aids.

\section{DISCUSSION}

All the subjects of this study derived benefit in hearing, due to the amplification in all the design-types of hearing aids. The results from table 2, 3, 4, 5 and 6 indicate that irrespective of the design-type incorporated in the hearing aid, the tinnitus improved with all the hearing aids. This corresponds to the findings of Searchfield et al. (2010) in their study titled 'Hearing aids as an adjunct to counseling', wherein they have employed the Tinnitus Handicap Questionnaire (THQ) ${ }^{5}$. The THQ studied the psychosocial behavior and tinnitus handicap. They found that the addition of hearing aids primarily for hearing impairment, led to significant reduction in Tinnitus handicap. However, that study did not compare between type of hearing aids or technical design settings. Also, Henry et al. $\left(2015^{5}\right)$ study dealt with hearing aids alone, had found hearing aid with special features provide significant benefit with respect to reducing the tinnitus affects ${ }^{6}$. The sound therapy devices were found to lower the tinnitus annoyance levels. Das et al. (2016) ${ }^{7}$ reported that hearing aids were the mainstay of tinnitus treatment as provided by audiology. There had been dissenting reports also. Celene McNeill et al. (2012) ${ }^{8}$ in their study on tinnituspitch masking, reported that despite use of hearing aids, there was no significant relief. In a similar study, Kochkin \& Tyler in 2008, reported that amplification did not provide effective relief of tinnitus ${ }^{9}$. In comparison to the above studies, our study comprised of a large sample of 108 subjects with severe and catastrophic degrees of tinnitus handicap, co-existing with at least a moderate degree of hearing impairment. The subjects were fitted with digitally programmable hearing aids, in a clinical setting, strictly conforming to standard and accepted guidelines. The three design types represent almost the entire variety of available newer technological design types. In hearing aid fitting, patient reported outcomes fare better than audiological testing. We employed an immensely popular and commonly used tool of patient-reported outcome viz. THI. The translated local language (Telugu) version facilitated better comprehension and thereby better reliability. Thus, our study would be relevant to day-today clinical practice. In our study, tinnitus improved in total and also in individual sub scale domains, with all the design types of hearing aid. When the benefit afforded by the individual design types were analyzed, the basic programmable group (D-Basic group) failed to help four subjects - three subjects with catastrophic degree handicap and one subject with severe degree of handicap i.e. a total of $11 \%$ failure. Similarly, in the digitally programmable hearing aids with tinnitus specific masking (DTS group), one subject with catastrophic degree of handicap, failed to show any mitigation of tinnitus. All the five above mentioned patients had to be given Tinnitus Retaining Therapy and fared better. Favorable differences were found between D-Basic \& DIM groups. However, the differences were not significant between DIM and DTS groups. The results also indicate that the DIM gives better benefit to client with hearing loss and tinnitus followed by DTS. This could be because tinnitus masker induces noise continuously. Our results are in congruence with that of Celene McNeill et al. (2012) ${ }^{8}$ who stated that results are obtained by masking and high frequency amplification in hearing aids which may be significant contributor in reducing tinnitus. Francis Kuk et al. (2010) ${ }^{10}$ stated that all the patients who used Hearing aid with added features such as Zen and Noise program options have reported $100 \%$ benefit in addressing tinnitus. This study also emphasized bilateral fitting in individuals with bilateral complaints. Similar findings were noted by Olaf Zagolski (2006) in their study on laterality of tinnitus and hearing aid fitting ${ }^{11}$ Sweetow et al. (2010) ${ }^{11}$ stated that delivery of fractal tones through hearing aids provides relief from tinnitus ${ }^{12}$. This method of treatment is called 'Zen' amplification method. However, they mentioned that Counseling and Cognitive Behavioral Therapy would also be necessary. Our results also correlated with the observations of Roeser \& Price $(1980)^{13}$ who found that 'when tinnitus is of a milder degree of handicap, total relief was observed more often. When the handicap is of a worse degree, only a partial relief was obtained.

\section{CONCLUSION}

The results of this study found that the advanced designs in the technology namely Basic amplification, tinnitus specific programming and inbuilt showed advantages over conventional digital hearing aid. Further, it was found that the technological advancements incorporated in the hearing aids provide better benefit in the tinnitus management. However, the point to be noted is that hearing aid treatment for tinnitus perception might not necessarily resolve tinnitus. But usage of hearing aids acts as an important component of tinnitus management. This study has proven the alternate hypothesis that usage of hearing aids reduces the distress of the tinnitus patients, in functional, emotional and catastrophic domains. Further the usage was found most effective in all the category of patients in all the age groups, onset, and nature of hearing loss and tinnitus and as well in the type of tinnitus. However, the drawbacks of the study show that the subjects with moderate or worse hearing loss and tinnitus were only recruited in the study. Only digital hearing aids were used in the study. Further, the hearing aids with greater number of channels and features may be used and studies may be done on specific variables and matched group of tinnitus patients. 


\section{ACKNOWLEDGEMENT}

The authors gratefully acknowledge the voluntary cooperation of the fellow audiologists and the cooperation of the subjects, in carrying out this large study. Dr Surya Prakasam Bandhakavi PhD, formerly Statistician in the National Institute for the Empowerment of Persons with Intellectual Disabilities, Secunderabad had been initially helping in the statistical analysis, until his untimely death. Subsequently, Dr. Raghunadha Acharya of Messrs. Data Kaisen continued the analysis. The authors gratefully acknowledge their erudite contributions.

\section{CONFLICT OF INTEREST}

The Author declares no potential conflict of interest on publishing this paper.

\section{REFERENCES}

1. McCormack A, Edmondson JM, Somerset S, Hall DA. systematic review of the reporting of tinnitus prevalence and severity. Hearing Research. 2016;337:70-9.

2. Newman CW, Jacobson GP, Spitzer JB. Development of the Tinnitus Handicap Inventory. Arch Otolaryngology Head Neck Surg. 1996; 122:143-8.

3. Songul A, Yezdan F, Reha R. The tinnitus Handicap Inventory: A study of validity \& Reliability. International Tinnitus Journal. 2007;13:94-8.

4. Rajendra Kumar P, Balakrishnan D. Translation of the Tinnitus Handicap Inventory (THI) into the Telugu language and Standardization. The International Tinnitus Journal. 2018;22:93-6.
5. Searchfield GD, Kaur M, Martin WH. Hearing aids as an adjunct to counseling: Tinnitus patients who choose amplification do better than those that don't. International Journal of Audiology. 2010;49:574-9.

6. Henry James A, Melissa F, Sara S, Susan G, Harvey Abrams. Validation of a Novel Combination Hearing Aid and Tinnitus Therapy Device. Ear \& Hearing. 2015;36:42-52.

7. Apratim D, Sinha AK, Akanksha K, Indranil C, Himanshu K. Effect of Using Amplification Device for Treatment of Tinnitus in Presbycusis in First Time Hearing Aid Users. IOSR Journal of Dental and Medical Sciences. 2016;15:49.

8. Celene Mc, Dayse T, Fadwa A, Searchfield GD, David W. Tinnitus pitch, masking, and the effectiveness of hearing aids for tinnitus therapy. International Journal of Audiology. 2012;51:914-9.

9. Kochkin S, Tyler R. Tinnitus treatment and the effectiveness of hearing aids hearing care professional perceptions. Hearing Review. 2008; 15: 14-8.

10. Francis K, Heidi P, Chi L. The efficacy of Fractal Music Employed in Hearing Aids for tinnitus management. Hearing review. 2010;1:1-6.

11. Olaf Zagolski K. Management of Tinnitus in Patients with Presbycusis. The International Tinnitus Journal. 2006;12:175-8.

12. Sweetow P, Robert W, Sabes K, Jennifer Henderson. An overview of common procedures for the management of tinnitus patients. Hearing Journal. 2010;63:11-12.

13. Roeser RJ, Price DR. Clinical experience with tinnitus maskers. Ear and Hearing. 1980;1:63-8. 